Vol. 23, No.4, pp.09-13, Year-2018

\title{
STUDY OF ENERGY LEVELS, QUADRUPLE ELECTRIC TRANSITION PROBABILITIES AND POTENTIAL ENERGY SURFACE FOR ISOTOPES 62-60 ZN USE IBM-1
}

\author{
Qaissar Abdulhussein Abbas and Aamer Mose Kadem \\ Educational Directorate of Qadisiyah, Iraq, Email caeer37@ gmail.com \\ Educational Directorate of Qadisiyah, Iraq, Email amer45@gmail.com
}

DOI: $10.29350 /$ jops.2018.23.4.906

\begin{abstract}
:
The aims of this study to the properties of the nuclear structure of isotopes light ( $62 \mathrm{Zn}, 60 \mathrm{Zn})$ using the first interactive boson model (IBM-1) Low energy levels of determination were determined by the type of dynamic symmetry of these isotopes ( $62 \mathrm{Zn}$, 60Zn) Similar to some of the energy levels that were not definitively identified and some were confirmed and the momentum and symmetry of others that were not previously identified in practice. Also, the surfaces of each nucleus were examined and nuclei were determined the symmetry.
\end{abstract}

KEYWORDS: potential energy surface, boson reaction IBM-1, energy levels, vibrational symmetry, Isotopes 62-60 Zn, nuclear structure.

\section{INTRODUCTION}

(Arima and Iachelo) proposed a new nuclear model called the IBM-1 interactive boson model. One of its objectives was to describe the vibrational and rotating spectra and intermediate states of the nuclei based on Group Symmetric instead of the geometric description of the kernel form variables The basic idea of the IBM-1 model assumes that the nuclei of even - even represent (core) of magic numbers, the nucleons outside the closed veneers duplicate with each other, creating what is called bosons, these bosons occupy one level the ground level and the level irritable, while the ground level and the momentum angular zero is benign boson type of $S$ ( $s$-boson) while the level irritated that takes momentum angular equal to 2 his bosons are of a kind of $d(d-$ boson) These levels are the two energies $\varepsilon_{s}, \varepsilon_{d}$ and respectively, and the difference between the energy tow boson $\varepsilon=\varepsilon \mathrm{d}-\varepsilon$ s often bosons of energy equal to zero type. The bosons with the same angular momentum and similar interact with each other, the total number of bosons $\mathrm{N}$ equals the number of type $\mathrm{s}$ bosons plus the number of type $d(N=n d+n s)$ a fixed number represents the number of duplication nucleons outside the closed veneers. [Arima and Iachello,1987] [Bonatsos, D,1988]

Many researchers have been studying the nuclear properties of isotopes of zinc in the rich light nuclei with neutrons area where the researcher [Lezell and Scott, 1974] studying the properties of low-lying energy levels by the dissolution of the gama rays. the researcher [Ekström and Fogelberg,1986] as studied the dissolution of isotopes ${ }^{75-80} \mathrm{Zn}$ characteristics and determined four-electrode-rich isotopes with neutrons . the researcher [Boucenn, 1990] study transitions in $\mathrm{Zn}$ and $\mathrm{Ni}$ isotopes depending on the specimen's crust and the study of wave-core analysis of the angular distribution. the researcher [Kenn,. 2002] studying the g-factor and the value of the transfer quad electric pole by the model of the crustthe researcher [Koizumi, and Seki, 2004] study excitement Alcolomah isotopes ${ }^{66-64} \mathrm{Zn}$ was determined account quadrant electrode and installation of ground packages by a model of interacting bosons and rotational symmetry model and calculation of surface energy effort by Nelson - Streunsky Nilsson-Strutinsky model. the researcher [Thomas et al, 2005] studying the nuclear structure of isotopes $\mathrm{Zn}$ and Ca-rich fission neutrons by ${ }^{238} \mathrm{U}$. The researcher . [Perru, 2006]study examining the polarization of isotopes ${ }^{74} \mathrm{Zn}$ and $70 \mathrm{Ni}$ and study the possibility of electrical transfer . the researcher [Vretenar and Meng,2011]studying the transformation of the forms the nucleus of the $\mathrm{Zn}$ isotopes with mass number less than 70 using shades of excitement and wave functions and determination quadrant electrode. the researcher [Gellanki, 2012]studying the higher twisting isotopes ${ }^{62} \mathrm{Zn}$. The researcher [Louchart ,2013] studying the collective nature of the low-lying levels of excitation energy to ${ }^{70,72,74} \mathrm{Zn}$ isotopes of half-life measured by AGATA spectrometer. the researchers [Sahuand Kota, 2015] studying forms of distortion in the isotopes ${ }^{70} \mathrm{Zn}$ and ${ }^{150} \mathrm{Nd}$ using crustal model. the [Pritychenko,2016] to study electrical and magnetic transitions and to determine the approximation value of the transmission of Gama

\section{THEORETICALI FOUNDATIONS}

For the purpose of describing the energies of a specific nuclear system, we need to influential Hamilton function for that system, Hamiltonian Operator of first interacting bosons model (IBM-1) including a double-edged for (One Body Interactions) and seven -edged for (One Body) and of( Two Body interactions) as follows [Iachello and Iscaker, 1991]: $\hat{H}=\varepsilon_{s}\left(\hat{s}^{\dagger} \cdot \hat{\tilde{s}}\right)+\varepsilon_{d} \sum_{m}\left(\hat{d}_{m}^{\dagger} \cdot \hat{\tilde{d}}_{m}\right)+\hat{W}$

$$
\hat{H}=\varepsilon_{s}\left(\hat{s}^{\dagger} \cdot \hat{\tilde{s}}\right)+\varepsilon_{d} \sum_{m}\left[\hat{d}_{m}^{\dagger} \cdot \hat{\tilde{d}}_{m}\right]
$$




\section{Al-Qadisiyah Journal Of Pure Science (QJPS)}

$$
\begin{aligned}
& +\sum_{L=0,2,4} \frac{1}{2} \sqrt{2 L+1} c_{L}\left[\left[\hat{d}^{\dagger} \times \hat{d}^{\dagger}\right]^{(L)} \times[\hat{\tilde{d}} \times \hat{\tilde{d}}]^{(L)}\right]_{0}{ }^{(0)} \\
& +\frac{1}{2} v_{0}\left[\left[\hat{d}^{\dagger} \times \hat{d}^{\dagger}\right]^{(0)} \times[\hat{\tilde{s}} \times \hat{\tilde{s}}]^{(0)}+\left[\hat{s}^{\dagger} \times \hat{s}^{\dagger}\right]^{(0)} \times[\hat{\tilde{d}} \times \hat{\tilde{d}}]^{(0)}\right]_{0}^{(0)} \\
& +\frac{1}{2} u_{o}\left[\left[\hat{s}^{\dagger} \times \hat{s}^{\dagger}\right]^{(0)} \times[\hat{\tilde{s}} \times \hat{\tilde{s}}]^{(0)}\right]_{0}^{(0)}+ \\
& u_{2}\left[\left[\hat{d}^{\dagger} \times \hat{s}^{\dagger}\right]^{(2)} \times[\hat{\tilde{d}} \times \hat{\tilde{s}}]^{(2)}\right]_{0}^{(0)}
\end{aligned}
$$

Where: $\varepsilon_{\mathrm{d}}, \varepsilon_{\mathrm{s}}$ represents the energy of $\quad$ (s-boson) and (dboson) respectively

$\mathrm{m}$ : Take the values $\mathrm{m}=0, \pm 1, \pm 2$, either $\hat{W}$ : represents (Boson-Boson Interaction), which represents $(\mathrm{L}=0,2) \mathrm{U}_{\mathrm{L}}$ and $\mathrm{V}_{\mathrm{L}}(\mathrm{L}=0,2)$ and $\mathrm{C}_{\mathrm{L}}(\mathrm{L}=0,2,4)$ the information's of (bosons interaction). The symbols within the brackets represent a duplication of angular momentum (Angular Momentum Coupling).

The first and second limits energies bosons - boson particle per reaction, while the other seven limits represents the energies of bosons- boson patrician one and two particles interaction. [Iachello and Iscaker, 1991]

You can formulate the equation (2) using a multi-polar expansion formula

$\hat{H}=\varepsilon \hat{n}_{d}+a_{o} \hat{P} \cdot \hat{P}+a_{1} \hat{L} \cdot \hat{L}+a_{2} \hat{Q} \cdot \hat{Q}+a_{3} \hat{T}_{3} \cdot \hat{T}_{3}+a_{4} \hat{T}_{4} \cdot \hat{T}_{4}$ ...... (3)

\section{DYNAMIC SYMMETY}

When applying the analytical solution on first interacting bosons model (IBM-1) there are only three cases can be analysed these three cases classified by three Group Chains star with Dynamic symmetry group $\mathrm{O}(6)$ and ending with $\gamma$-Unstable Dynamical Symmetry $(\gamma$-Unstable Limit [Iachello and Iscaker, 1991] [Bonatsos, D, 1988]:

$\mathrm{U}(6)$

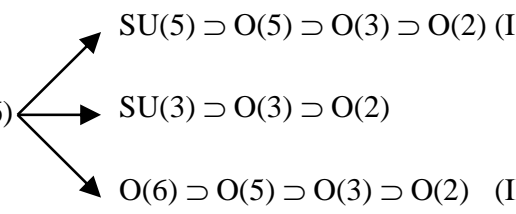

..(4)

The above equation represents the three species in the energy selections are bosons $(\varepsilon)$ is much greater than the effort interaction between the bosons, which means that $(\varepsilon$ > V) called this limitation specifically vibratory, either limitation (II III) to occur when energy bosons are much larger than interaction between the bosons effort and when the four-pole torque reaction occurs (QQ) between bosons and called specifically rotational this selection, the duplex interaction ( $\hat{\mathbf{P}} . \hat{\mathbf{P}}$ ) happening between bosons is dominant relative to the energy bosons and called this selection select Kama unstable ( $\gamma$-unstable) O (6 ).

The three cases can be summarized as follows:

\subsection{VIBRATIONAL} (VIBRATIONA

DYNAMIC

SYMMETY LIMIT)

This symmetry is described by the subgroup SU(5) Which can be written with their quantitative numbers as follows[Iachello and Iscaker, 1991] :

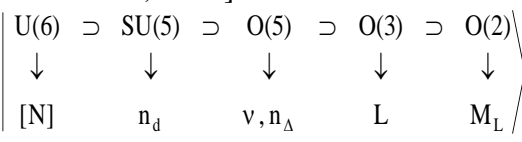

\subsection{DYNAMIC ROTTIONAL SYMMETY}

This is called sub-described symmetry group SU (3) and which can be writes with quantitative numbers as follows [Iachello and Iscaker, 1991] :

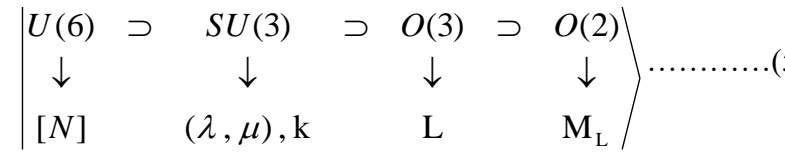

\section{3. $\gamma$-UNSTABLEDYNAMICSYMMETY}

\section{( $\gamma$-U UNSTABLEDYNAMIC LIMIT $)$}

This symmetry is described as subgroup $\mathrm{O}$ (6) which can be written with its quantitative numbers as follows:

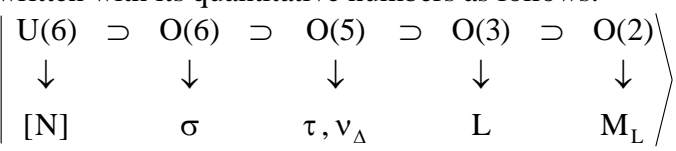

\section{RESULTS AND DISCUSSION}

In this study we studied a set of neutron pairs of $\mathrm{N}=30$ to $\mathrm{N}=$ 32 and a number of protons equal to $30\left({ }^{62} \mathrm{Zn},{ }^{60} \mathrm{Zn}\right)$. When studying the nuclear properties of this series of mass isotopes (60-62) Determine the behaviour of each counterpart depending on the theoretical results calculated using the IBM-1 interactive boson model and compare them with the available practical values as low energy levels with positive symmetry In the series of matrilineal isotopes in the series of conjugal isotopes in which the number of neutrons of cortex 50, which is a nucleus of collective properties Use the IBM-code program to calculate the power levels of the isotope series where equation coefficients (3) Of process diagrams for these cores table (1) Represents the number of bosons and transaction values used in the IBM-1 model that gave the best correlation between theoretical and practical energy levels.

Table (1) shows the parameter values used in the program IBM-1 code

\begin{tabular}{|l|l|l|l|l|l|l|l|}
\hline Parameters & \multirow{N}{*}{$\mathrm{N}$} & $\varepsilon \mathrm{s}$ & $\varepsilon \mathrm{d}$ & $\mathrm{A} 1$ & $\mathrm{~A}_{2}$ & $\mathrm{~A}_{3}$ & $\mathrm{~A}_{4}$ \\
\cline { 1 - 8 } Isotones & & & & & & & \\
\hline${ }^{60} \mathrm{Zn}$ & 10 & 0.00 & 1.042 & 0.00 & 0.00 & 0.00 & 0.00 \\
\hline${ }^{62} \mathrm{Zn}$ & 9 & 0.00 & 0.956 & 0.00 & 0.00 & 0.00 & 0.00 \\
\hline
\end{tabular}

The theoretical values of the energy levels calculated by the IBM-1 program for these isotopes are illustrated in Figures (2) where they were compared with the practical values and were highly consistent.

In addition, angular momentum and similarity of certain energy levels that were not clearly defined have been predicted and some of them have been confirmed and momentum has been created and some others have not been identified in practice as in Tables (2-3). 


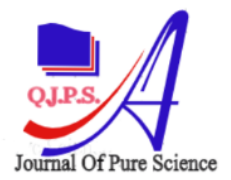

\section{Al-Qadisiyah Journal Of Pure Science (QJPS)}

The values of the coefficient $(\varepsilon)$ representing the energy of the bosons decrease with the increase in the number of neutrons, and the reduction values in equation (3) represent the Pairing Interactions in the Hamilton effect. This limit helps to study the nuclei, which is characterized by the type of packages with the package ( $\gamma$-band), which is lower than those packages of the type ( $\beta$-band) and this $\left(\varepsilon / a_{0}\right)$ will decrease with the increase in the number of neutrons. The limit $(\varepsilon)$ is the main parameter of determination SU (5) And $\left(\mathrm{a}_{0}\right)$ is the primary coefficient of determination $\mathrm{O}(6)$ That the value of the coefficient $\varepsilon$ is dominant in the isotope ${ }^{60-}$ ${ }^{62} \mathrm{Zn}$ That is to force of the reaction of the bosons is dominant and the shape of the nuclei tends to be vibrational, noting that there is a ratio of the effect of the coupling factor $\left(\mathrm{a}_{0}\right)$ in the two nuclei ${ }^{62-60} \mathrm{Zn}$.
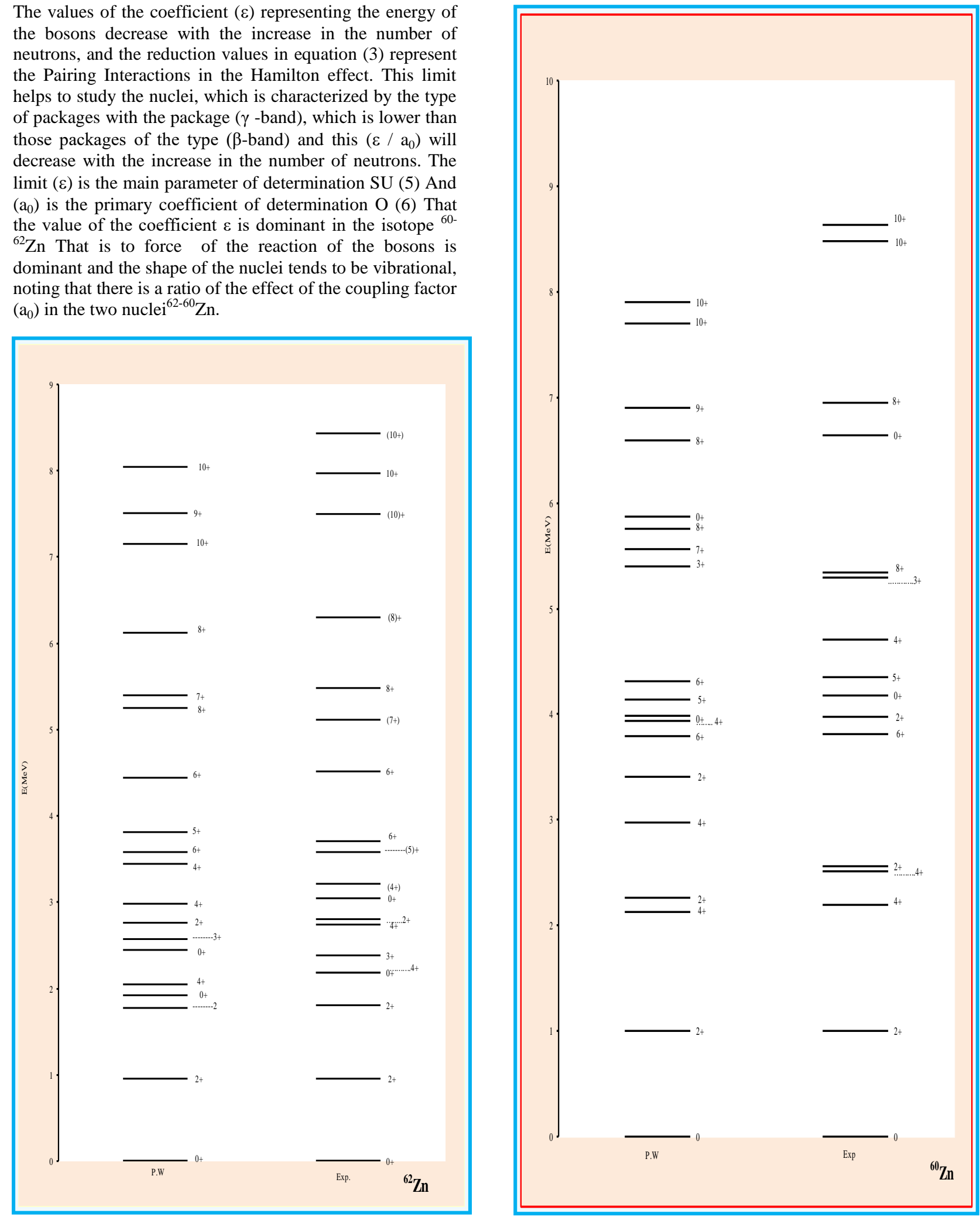

Formats (1) shows a comparison between the theoretical energy levels of the isotopes studied and calculated by the IBM-1 model with the corresponding process values. 
Table (4) Comparison of energy levels theoretical and practical to isotopes $62 \mathrm{Zn}$

\begin{tabular}{|c|c|c|c|}
\hline $\mathrm{J} \pi$ & $\begin{array}{c}\text { Current } \\
\text { results }\end{array}$ & $\begin{array}{l}\text { The } \\
\text { practical } \\
\text { value }\end{array}$ & Notes \\
\hline $0+1$ & 0 & 0 & \\
\hline $2+1$ & 0.953 & 0.953 & \\
\hline $4+1$ & 2.186 & 2.047 & \\
\hline $6+1$ & 0.578 & 0.582 & \\
\hline $8+1$ & 5.481 & 5.346 & $\begin{array}{l}\text { Energy level has been confirmed } \\
\left(\begin{array}{lll}(5.481 & \mathrm{Mev}) \text { by spin and } \\
\text { symmetry }(8+1)\end{array}\right.\end{array}$ \\
\hline $0+2$ & 2.341 & 2.286 & \\
\hline $2+2$ & 1.804 & 1.77 & \\
\hline $2+3$ & 2.803 & 2.758 & \\
\hline $3+1$ & 2.384 & 2.57 & \\
\hline $4+2$ & 2.743 & 2.985 & \\
\hline $4+3$ & 3.209 & 3.445 & \\
\hline $10+1$ & 7.5 & 7.35 & $\begin{array}{l}\text { Energy level has been confirmed } \\
(7.5 \mathrm{Mev}) \text { by spin and symmetry } \\
(10+1)\end{array}$ \\
\hline $5+1$ & 3.586 & 3.715 & $\begin{array}{l}\text { Energy level has been confirmed } \\
(3.586 \mathrm{Mev}) \text { by spin and } \\
\text { symmetry }(5+1)\end{array}$ \\
\hline $6+2$ & 4.515 & 4.438 & \\
\hline $7+1$ & 5.143 & 5.245 & $\begin{array}{l}\text { Energy level has been confirmed } \\
\left(\begin{array}{lll}5.143 & \text { Mev }) \text { by spin and } \\
\text { symmetry }(7+1)\end{array}\right.\end{array}$ \\
\hline $8+2$ & 6.3 & 6.126 & $\begin{array}{l}\text { Energy level has been confirmed } \\
(6.3 \mathrm{Mev}) \text { by spin and symmetry } \\
(8+2)\end{array}$ \\
\hline $0+3$ & 3.042 & 2.846 & $\begin{array}{l}\text { Energy level has been confirmed } \\
(3.042 \mathrm{Mev}) \text { by spin and } \\
\text { symmetry }(0+3)\end{array}$ \\
\hline $9+1$ & 7.976 & 7.706 & 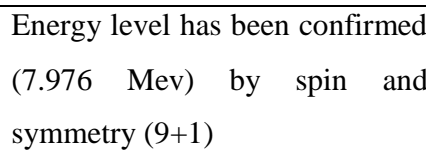 \\
\hline $10+2$ & 8.437 & 8.048 & $\begin{array}{l}\text { Energy level has been confirmed } \\
(8.437 \mathrm{Mev}) \text { by spin and } \\
\text { symmetry }(10+2)\end{array}$ \\
\hline
\end{tabular}

\section{POTENTIAL ENERGY SURFACE}

Table (3)Comparison of energy levels theoretical and practical to isotopes $60 \mathrm{Zn}$

\begin{tabular}{|c|c|c|c|}
\hline $\mathrm{J} \pi$ & $\begin{array}{l}\text { Curren } \\
\mathrm{t} \\
\text { results }\end{array}$ & $\begin{array}{l}\text { The } \\
\text { practical } \\
\text { value }\end{array}$ & Notes \\
\hline $0+1$ & 0 & 0 & \\
\hline $2+1$ & 1.003 & 1.003 & \\
\hline $4+1$ & 2.193 & 2.13 & \\
\hline $6+1$ & 3.808 & 3.755 & \\
\hline $8+1$ & 5.291 & 5.351 & \\
\hline $0+2$ & 4.18 & 3.997 & $\begin{array}{l}\text { Energy level has been confirmed } \\
(4.18 \mathrm{Mev}) \text { by spin and symmetry } \\
(0+3) \text { which was unconfirmed } \\
(0+, 2+)\end{array}$ \\
\hline $2+2$ & 2.558 & 2.245 & \\
\hline $2+3$ & 3.972 & 3.811 & \\
\hline $3+1$ & 5.337 & 5.401 & $\begin{array}{l}\text { Energy level has been confirmed } \\
(5.337 \mathrm{Mev}) \text { by spin and } \\
\text { symmetry }(0+3) \text { which was } \\
\text { unconfirmed }(3+, 4+)\end{array}$ \\
\hline $4+2$ & 3.51 & 2.659 & $\begin{array}{l}\text { Energy level has been confirmed } \\
(3.51 \mathrm{Mev}) \text { by spin and symmetry } \\
(0+3) \text { which was unconfirmed } \\
(2+, 4+)\end{array}$ \\
\hline $4+3$ & 4.71 & 4.538 & 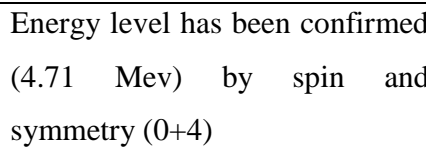 \\
\hline $10+1$ & 8.475 & 8.303 & \\
\hline $5+1$ & 4.199 & 4.145 & $\begin{array}{l}\text { Energy level has been confirmed } \\
(4.199 \mathrm{Mev}) \text { by spin and } \\
\text { symmetry }(5+1)\end{array}$ \\
\hline $6+2$ & ------ & 4.315 & It has been expected \\
\hline $7+1$ & ------ & 5.562 & It has been expected \\
\hline $8+2$ & 6.95 & 6.994 & \\
\hline $0+3$ & 6.639 & 5.9702 & \\
\hline $9+1$ & & 6.9047 & It has been expected \\
\hline $10+2$ & 8.636 & 8.902 & $\begin{array}{l}\text { Energy level has been confirmed } \\
(8.636 \mathrm{Mev}) \text { by spin and } \\
\text { symmetry }(10+2)\end{array}$ \\
\hline
\end{tabular}




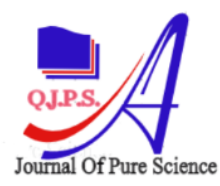

\section{Al-Qadisiyah Journal of Pure Science (QJPS)}

Vol. 23, No. 4, pp.09-13, Year-2018

Potential Energy Surface is calculated after determining the parameters of the Hamilton function for each nucleus shown in Table 1. Table 2 shows the coefficients used in the (PES for $t)$ he calculation of the surface energy of $\mathrm{V}(\mathrm{N}, \beta$, ).

Table (1) shows the parameter values used in the program IBM-1

\begin{tabular}{|l|l|l|l|l|l|l|l|}
\hline Parameters & $\mathrm{N}$ & $\varepsilon \mathrm{s}$ & $\varepsilon \mathrm{d}$ & $\mathrm{A} 1$ & $\mathrm{~A}_{2}$ & $\mathrm{~A}_{3}$ & $\mathrm{~A}_{4}$ \\
\cline { 1 - 5 } Isotones & & & & & & & \\
\hline${ }^{60} \mathrm{Zn}$ & 10 & 0.00 & 1.042 & 0.00 & 0.00 & $\begin{array}{l}0.0 \\
0\end{array}$ & $\begin{array}{l}0.0 \\
0\end{array}$ \\
\hline${ }^{62} \mathrm{Zn}$ & 9 & 0.00 & 0.956 & 0.00 & 0.00 & $\begin{array}{l}0.0 \\
0\end{array}$ & $\begin{array}{l}0.0 \\
0\end{array}$ \\
\hline
\end{tabular}

Table (2) shows the parameter values used in the program IBM-1 code Isotopes used in the research

\begin{tabular}{|l|l|l|l|l|l|l|l|}
\hline Parameters & $\mathrm{N}$ & $\varepsilon_{\mathrm{PS}}$ & $\hat{\mathrm{P}} . \hat{\mathrm{P}}$ & $\hat{\mathrm{L}} . \hat{\mathrm{L}}$ & $\hat{\mathrm{Q}} . \hat{\mathrm{Q}}$ & $\hat{\mathrm{T}}_{3} \cdot \hat{\mathrm{T}}_{3}$ & $\hat{\mathrm{T}}_{4}, \hat{\mathrm{T}}_{4}$ \\
\hline${ }^{\text {Isotones }}$ & & & & & & & \\
\hline${ }^{60} \mathrm{Zn}$ & 10 & $\mathbf{0 . 9 4 6}$ & $\mathbf{0 . 0 0 0 3}$ & $\mathbf{0 . 0 1 6 0}$ & $\mathbf{- 0 . 0 0}$ & $\begin{array}{l}\mathbf{0 . 0} \\
\mathbf{0}\end{array}$ & $\mathbf{0}$ \\
\hline${ }^{62} \mathrm{Zn}$ & 9 & $\mathbf{0 . 8 6 9}$ & $\mathbf{0 . 0 0 1 0}$ & $\mathbf{0 . 0 1 5 3}$ & $\mathbf{- 0 . 0 0}$ & $\begin{array}{l}\mathbf{0 . 0} \\
\mathbf{0}\end{array}$ & $\mathbf{0}$ \\
\hline
\end{tabular}

While Potential Energy Surface gives the final shape of the nucleus that corresponds to the Hamilton function and the two variables $\beta$ and $\gamma$ as Represent $\beta$ Keep away from the spherical effort of the nucleus, which is a measure of total deformation when it is $\beta=0$ The shape of the nucleus is spherical and when it is $\beta \neq 0$ The shape is distorted As for the variable $\gamma$ It represents the amount of deviation from the axis of symmetry and is closely related to the nucleus when it is $\gamma={ }^{\circ} 0$ The shape is elongated in the direction of the poles And when they are $\gamma={ }^{\circ} 60$ The nucleus takes a flattened shape at the poles.

That shapes from (1) The axial symmetry and contour lines of the axial non-symmetric angles of the calculated Potential Energy Surface of nanotubes under study, Where the Potential Energy Surface was drawn as a function of deformation $(\beta=0 \rightarrow 2.4$ ) Where we note that the minimum value of Potential Energy Surface for Nucleus $\left({ }^{60} \mathrm{Zn},{ }^{62} \mathrm{Zn}\right)$ Equal $\quad(-0.102$ and -0.192$)$ It is located at $\beta$ $=0$ With axial symmetry for $\gamma={ }^{\circ} 0$ and $\gamma={ }^{\circ} 60$ Which indicates the presence of some vibrational qualities in addition to the qualities of nuclei $\mathrm{O}(6)$ Where the smallest value for $\beta$ Specifically, $S U$ (5) is equal to 0 This is confirmed by the contouring of these nuclei, where they are concentric circles That is, the nuclei have superficial vibrations around the spherical shape and do not possess the characteristics of the nuclei.

Through the results we reached conclusion:

The effect of the increase in the number of neutrons on nuclear power levels in the determination of the location of isotopes ${ }^{60-62} \mathrm{Zn}$ of the three selections SU (5), SU (3) and O (6) shows that the current study results show that $60-62 \mathrm{Zn}$ The transition zone for the determinations SU (5) and O (6). The IBM-1 engineering model was able to accurately describe the equal voltage surfaces of the nuclei under study and determine the shape of the surface vibrations and the shape of the rotational nuclei. That the nuclei in the studied isotopes, the nuclei suffer superficial vibrations around the spherical shape and do not possess the characteristics of the nuclei.

\section{REFERENCES}

Arima, A. and Iachello, F. (1987). P.3. " The Interacting Boson Model", The Syndicate Press of the University of Cambridge,England.

Bonatsos, D. (1988). P.102 " Interacting Boson Model of Nuclear Structure" Oxford University Press, New York.

Boucenn, A.(1990). " Transfer reactions on even Ni and Zn isotopes ". Physical Review C. Vol42. P1297.

Browne, E. and Tuli, J. (2010). Nuclear Data Sheetshttps://www-nds.iaea.org.Vol 111,P1093 .

Ekström, B. and Fogelberg, B. (1986). " Decay Properties of 75-80Zn and $\mathrm{Q} \beta$-values of Neutron-Rich $\mathrm{Zn}$ and $\mathrm{Ga}$ Isotopes ". Physica Scripta .Vol 34 . P6.

Gellanki,J. et al.(2012). "High-spin structure studies in 62Zn".Physica Scripta.Vol 150. P 113.

Iachello, F. and Iscaker K., (1991) P.29."The Interacting Boson Fermion Model "CambrigeUniversityPress, Cambrige,

Kenn, K. et al. (2002). " Measurements of $g$ factors and lifetimes of low-lying states in 62-70Zn and their shell model implication". Phys. Rev. C.Vol65. P 343

Koizumi,M. and Seki,H . (2004)." Coulomb excitation of stable even-even $\mathrm{Zn}$ isotopes ". Japan Atomic Energy Research Inst. Vol36. P44(2004).

Lezell, R.. and Scott, H. .(1974). "The $\gamma$-ray decay properties of low-lying levels of ${ }^{65} \mathrm{Zn}$ via the ${ }^{65} \mathrm{Cu}(\mathrm{p}, \mathrm{n} \gamma) 65 \mathrm{Zn}$ reaction ". Nuclear Physics A. Vol 218. P470.

Louchart, C. et al .(2013)" Collective nature of low-lying excitations in 70,72,74Zn from lifetime measurements using the agata spectrometer demonstrator".Phys.Rev.Vol87.P10.

Mccutchan, E(2012).Nuclear Data Sheets https://wwwnds.iaea.org.Vol 113, P1735 .

Perru, O. et al .(2006)." Enhanced Core Polarization in Ni70 and Zn74".Phys. Rev.Vol96. P 232501

Pritychenko, B. et al (2016)."An Update of B(E2) Evaluation for Transitions in Even-Even Nuclei near".N Z 28". Phys. Rev. C.Vol69. P 8.

Sahu, R. and Kota, V .(2015)." Shape effects on double beta decay of $70 \mathrm{Zn}$ and $150 \mathrm{Nd}$ in deformed shell model". INSPIRE.Vol 1501. P07674.

Singh, B. (2007). Nuclear Data Sheets. https://wwwnds.iaea.org.Vol 108,P 197

Thomas, J. et al. (2005)." Nuclear structure studies of neutronrich $\mathrm{Cu}$ and $\mathrm{Zn}$ isotopes produced by means of proton-induced fission of $238 \mathrm{U} "$. Academic Journal.Vol798. P131.

Vretenar, S.and Meng,D.(2011)." Microscopic analysis of spherical to F-soft shape transitions in zn isotopes". Science China Physics, Mechanics and Astronomy .Vol54. P 224 